\title{
Why our best theories of perception and physics undermine realism
}

\author{
Michael H. Herzog ${ }^{1}$ \& Adrien Doerig ${ }^{2}$ \\ ${ }^{1}$ Laboratory of Psychophysics, Brain Mind Institute, École Polytechnique Fédérale de Lausanne (EPFL), Switzerland \\ ${ }^{2}$ Donders Institute for Brain, Cognition and Behaviour, Radboud University, Nijmegen, The Netherlands
}

\begin{abstract}
Realism about objects proposes an isomorphism between the objects of the external world and the corresponding mental representations. A candle on a table leads to the percept of this candle. Ontologically, realism locates objects between the entities of fundamental physics and the perceiver. Here, we show that under the assumption of a mind independent world of physical particles there is no space for a mind independent world of objects. The mental representations partition the physical world into classes of states, each class corresponding to one mental representation. Since there are many more physical states than representations, the mapping is not unique. Hence, different perceivers may maintain different partitions of the physical world, leading to different mind dependent representations and objects. In general, we show that if there is one fundamental ontology (fundamental physics), one cannot have any other ontology (objects) in addition. Furthermore, we show that realism is not even a desirable goal of perception. These considerations by no means favor dualism or solipsism. These ontological considerations are presented in part II. Based on neuroscience results, we show in part I that realism is not an epistemologically tenable position either.
\end{abstract}

\section{Introduction}

\section{Realism in perception}

According to direct realism, we perceive the world as it is. Encountering an object leads to a percept of exactly this object (figure 1a). Perception provides ground truth (Reid, 1764). Realism is grounded in the everyday life experience that we can trust our senses. Objects are simply there. In naïve or direct realism, the sensory representation provides ground truth. Indirect realism proposes that the sensory representation does not provide ground truth but later stages of processing do so. Both types of realism subscribe to an isomorphic, one-to-one correspondence between objects of the external world and mental representations.

\section{Anatomy and physiology of the visual system}

Light (luminance) enters the optical apparatus and hits the retina where photons activate photoreceptors (Figure 1b). Photoreceptors convert the energy of the light into electrical signals. In subsequent, hierarchical stages, the signals of the photoreceptors are further processed and features are extracted. For example, in primary visual cortex V1, the first cortical processing stage, lines and contours are extracted. Neurons in higher visual areas, such as the inferior temporal cortex, code for objects and faces. In neuroscience, isomorphisms are proposed between elements of the external world and neural representations in the brain. For example, a 
line in the outer world is detected by neurons sensitive to lines in V1, which is the basis of the perception of the line. In the spirit of philosophical eliminativism, subjectivity can by eliminated since percepts are isomorphic to their neural representations (Churchland \& Churchland, 1998).

\section{Part I: Perception, Realism \& Truth conditions}

First, we will present uncontroversial facts of vision science, which show that, even if there were a mind independent world of objects, it must be doubted whether we could perceive these objects as they truly are. Some of the arguments and their implications are well known (Hardin, 1993; Hoffman, Singh, \& Mark, 2013; Koenderink, 2014; Mausfeld, 2015; Lotto, 2017), others are new. In part II, we will challenge the ontological status of objects as mind independent entities.

\section{Sensation la: The bottleneck}

Visual processing starts at the retina where photoreceptors convert light into electrical signals. The human visual system usually contains only three types of photoreceptors (blue, green, and red), which are sensitive to light in the range from 400 to $700 \mathrm{~nm}$. Objects reflecting light of wavelengths outside this range, such as UV-light, are invisible.

Photoreceptors of other animals show different characteristics. For example, honeybees also have three photoreceptors, which are sensitive to light in the range from 300 to $650 \mathrm{~nm}$ including the ultraviolet range reflected by many flowers. Different sensory systems represent the world differently.

Conclusion: There is no 1-1 correspondence between objects and mental representations because not all (properties of) objects are represented.

\section{Sensation Ib: Metamers}

Even within the visible range of light, perception is by far not fully representing the richness of the external world. Light is usually not monochromatic, i.e., consisting of one wavelength only, but is a composite of many wavelengths (figure 1c). For example, white light contains photons of all wavelengths of the visible light. Because humans are equipped with only three photoreceptors, they cannot discriminate between so-called metamers, i.e., different composites of light leading to the same colour percept. Only for a creature with infinitely many different photoreceptors, metamers would not exist, i.e., there need to be infinitely many photoreceptors for each position of the visual field. Metamers are not restricted to color, they are ubiquitous in perception (Freeman \& Simoncelli, 2011).

Conclusion: Different objects may appear identical because of metamers. There is no 1-1 correspondence between objects and mental representations. 
a)

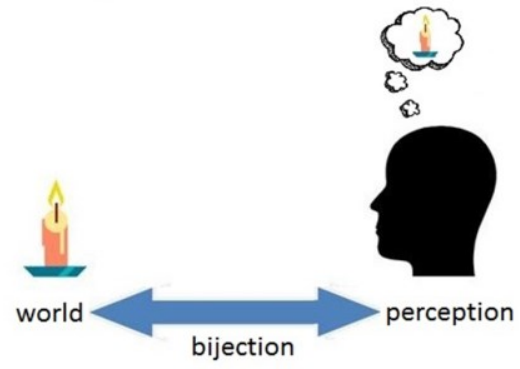

c)

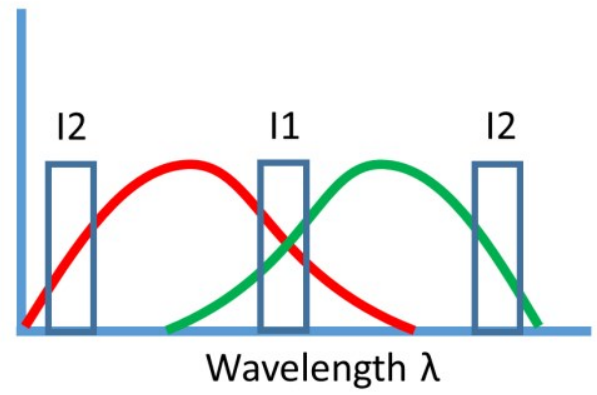

b)

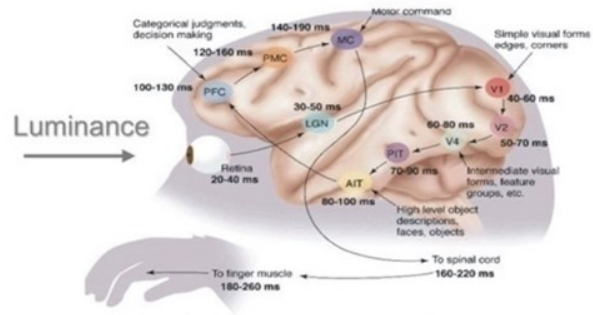

d)

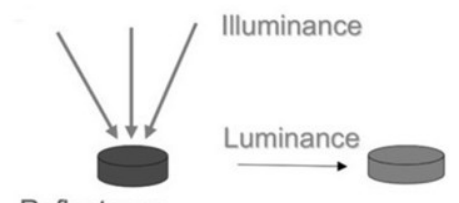

Reflectance e)

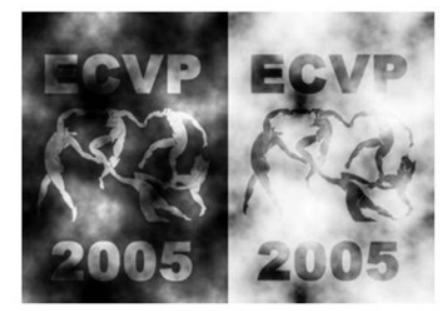

f)

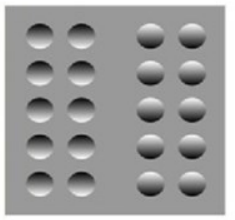

g)

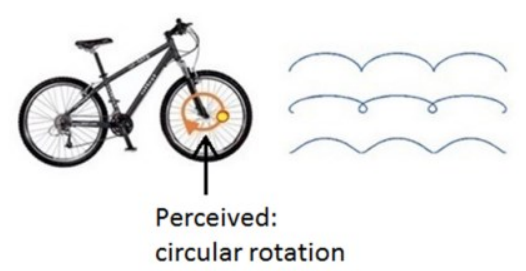

Figure 1. a) Realism and the world-perception isomorphism. According to realism, there is a 1-1 correspondence (or bijection) between objects in the world and the corresponding mental representations. For example, there is a candle in the outer world causing the percept of a candle. b) The visual system. Vision starts at the retina and continues in subsequent cortical areas. Neurons in early visual areas (V1, V2) code for basic features, such as edges and lines, whereas neurons in higher cortical areas (PIT, AIT) are sensitive to entire objects (from Thorpe \& Fabre-Thorpe, 2001). c) Perceptual metamers: The $x$-axis shows the wavelength of light. The y-axis shows how strongly a photoreceptor responds to a given wavelength. For example, the "red"-photoreceptor responds most strongly to monochromatic light of a frequency of about $560 \mathrm{~nm}$ and to lesser degrees to frequencies deviating from this peak frequency. Metamers are stimuli that are physically different but are perceptually indistinguishable from each other. For example, the object I1 reflects monochromatic light of only medium wavelength, exciting red and green photoreceptors equally. The second object 12 emits light of two wavelengths. The colours of both 
objects appear as identical because the output of the photoreceptors is identical. In this example, we restricted ourselves to two photoreceptors as they are present in dichromats but the same principles apply also with three and more photoreceptors. d) Vision is ill-posed. The light that enters the eye (luminance $L$ ) is the product of the light shining on an object (illuminance I) and the material properties of the object (reflectance $\mathrm{R}$ ): I $\times \mathrm{R}=\mathrm{L}$. There are infinitely many combinations of I and $\mathrm{R}$ leading to the very same luminance $L$. Hence, a single photoreceptor cannot "tell" whether a banana is yellow because of the material properties $(R)$ or of the illuminance (I). Almost all visual aspects, including size, 3D, and motion perception, are ill-posed. e) Colour constancy: The luminance of each pixel corresponding to the dancers is the same in the two images. The appearances of the dancers differ so strongly because the perceived colour of a point in the image is strongly influenced by the context (Anderson \& Winawer, 2008). f) Priors: The left and right parts of the image are identical except that the second picture is flipped by 180 degrees. On the left, craters are seen, bumps are seen on the right. The illusion is usually explained by the fact that humans have a prior that the sun light comes from overhead. g) Vision is reference dependent. The reflector on a wheel of a bike appears to rotate on a circular orbit but it is cycloidal when determined in Euclidean, external world coordinates. We cannot see the cycloidal motion because we perceive the reflector motion with respect to the moving bike (test yourself: which of the three blue trajectories is the "veridical" one?). We perceive the circular motion because we subtract the horizontal bike motion from the cycloidal motion. What is the veridical reference frame, the bike or the external world? In general, there are zillions of reference systems in real world situations.

\section{Perception I: III-posed problems}

Almost all types of visual processing are ill-posed. For example, the light hitting the photoreceptors, the luminance $(L)$, is a product of the light shining on an object, the illuminance $(\mathrm{I})$, and the material properties of the object, the reflectance ( $R$; figure $1 \mathrm{~d})$ :

Luminance $=$ Illuminance $*$ Reflectance

Obviously, there is no unique solution for this equation because there are two unknowns ( $\mathrm{I}$ and $\mathrm{R}$ ) and only one known variable (L). In fact, the very same object can reflect infinitely many luminances, depending on the Illuminance. Hence, the Luminance, and therefore photoreceptor activity, can by no means provide ground truth about the colour/reflectance of an object. The first stage of information processing is the stage where the fewest information about the object properties is available. To solve the ill-posed problems of vision, perception relies not only on context but also strongly on priors, as nicely demonstrated by the crater illusion (Figure 1f). Only when prior knowledge and contextual information are taken into account, it is possible to estimate the "veridical" reflectance of the object (so called colour constancy). Still, under certain conditions, colour constancy is limited (figure 1e). Colour is just one example for an ill-posed problem. Size, motion, lighting, 3D and shape perception are all ill-posed problems. The external world is 3-dimensional (string theory notwithstanding) but the retina is $2 \mathrm{D}$. Without priors, it is in principle impossible to infer the third dimension from a 2D representation.

Conclusions: Again, there is no 1-1 correspondence between objects and mental representations because the computation of the properties of objects is a mathematically unsolvable, ill-posed problem. The photoreceptor level is the worst location for ground truth. Hence, direct realism cannot be true. III-posed problems can only be "approximately solved" by the brain when context and priors are used. 


\section{Perception II: The reference frame}

Motion perception is always relative to a reference. The motion trajectory of a reflector on a bike wheel appears to be circular. However, it is cycloidal in a Euclidian reference frame centred on the observer (figure 1g). We perceive circular motion because we subtract the horizontal motion of the bike from the reflector motion. Obviously, there is no unique "true" motion trajectory. Different reference frames lead to different percepts and there can be zillions of reference frames. Similar compelling demonstrations are found here: http://www.youtube.com/watch?v=1F5ICP9SYLU.

Conclusion: Again, there is no 1-1 correspondence between objects and mental representations, because perception depends on reference frames. There are zillions of reference frames and no one is "truer" than the others. The very same object may appear in many facets, challenging realism conceptually.

\section{Objects I: Extraction \& representations}

In realism, the presentation of a candle is thought to lead to the perception of this candle because the corresponding mental representation is activated by the presence of the candle (figure 1a). However, this presupposes that the corresponding representation exists at all. In fact, missing holistic representations lead to failures of object recognition. For example, a lesion in the fusiform face area (FFA) can lead to a loss of the perception of individual faces whereas the recognition of the contours of the faces is still intact since low level processing is unaffected (Tanaka \& Farah, 1993). A similar effect can be experienced in figure 2a. Humans easily spot the spatial misconfiguration of the eyes and mouth of Margaret Thatcher in the right image with the upright face but hardly when the images are rotated (left image; Thompson, 1980) because we have holistic, spatial representations for upright facial configurations but not for up-side down faces. If humans had similar representations for inverted faces, we would spot the spatial oddities right away. The very same considerations hold true for all sorts of objects.

Hence, even when photoreceptor activities are identical for two perceptual systems, perception may strongly differ depending on whether or not representations exist. If there is no face representation, faces are invisible. If there are no line representations, lines are invisible. The photoreceptors host all the incoming visual information but there are no "objects" at this level. Objects need to be extracted. Similarly, a projection screen has all the information needed to extract objects, but no means to do so.

As a further illustration, in figure $2 \mathrm{~b}$ (left), the face of Kant is easily spotted. On the right, the luminance values are shown as numbers. It is not easy to spot Kant from the numbers because humans have no detectors for extracting structure from numbers, even though the image of Kant and the number representation are fully equivalent in terms of the information provided. What matters is whether the information can be extracted, i.e., can be computed. There is a double dissociation: the number representations in every other column have integer values, but this structure is not visible in the image of Kant because humans are not sensitive to small luminance changes. 
a)
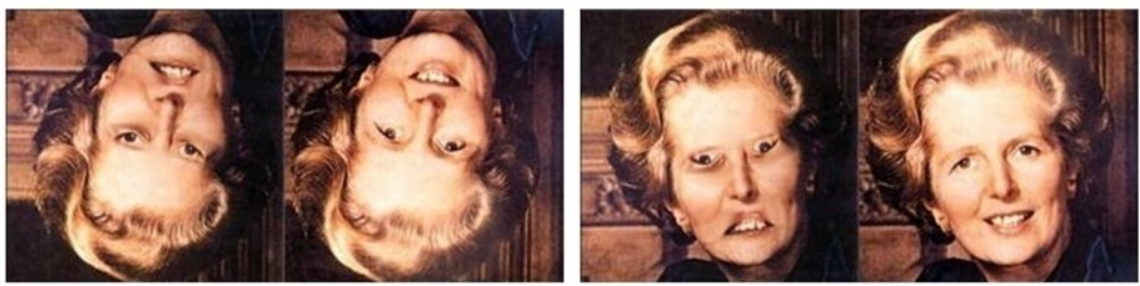

b)

$\begin{array}{ccccc}20.81472 & 27 & 26.70936 & 23 & 24.56882 \\ 27.90579 & 26 & 23.75469 & 26 & 30.46939 \\ 29.12699 & 26 & 24.27603 & 27 & 29.0119 \\ 24.91338 & 28 & 28.6797 & 25 & 23.33712 \\ 20.63236 & 30 & 28.6551 & 21 & 26.16218 \\ 22.09754 & 28 & 23.16261 & 21 & 36.79428 \\ 23.2785 & 24 & 19.119 & 22 & 40.31122 \\ 21.54688 & 21 & 19.49836 & 23 & 36.52853 \\ 25.95751 & 25 & 22.95974 & 25 & 31.16565 \\ 23.96489 & 23 & 21.34039 & 24 & 30.60198 \\ 22.15761 & 22 & 21.58527 & 24 & 30.26297 \\ 24.97059 & 24 & 22.22381 & 26 & 32.65408\end{array}$

$\begin{array}{lllllll}23 & 21.40181 & 24 & 26.48679 & 37 & 59.08552 & 68 \\ 25 & 22.07597 & 28 & 40.43586 & 41 & 58.26248 & 75 \\ 24 & 29.23992 & 45 & 58.44678 & 53 & 66.80101 & 86 \\ 25 & 41.12332 & 65 & 61.30635 & 62 & 77.02922 & 94 \\ 37 & 52.18391 & 65 & 49.50851 & 57 & 74.92885 & 94 \\ 53 & 55.23995 & 52 & 45.51077 & 51 & 66.73033 & 90 \\ 54 & 55.41727 & 53 & 57.81763 & 58 & 70.48861 & 95 \\ 46 & 55.04965 & 68 & 68.79483 & 69 & 82.57853 & 107 \\ 33 & 42.90272 & 57 & 99.64432 & 107 & 96.23728 & 126 \\ 32 & 41.94479 & 58 & 129.3786 & 151 & 155.4588 & 180 \\ 33 & 47.49086 & 69 & 129.8116 & 165 & 181.9631 & 201 \\ 39 & 62.48925 & 91 & 128.5328 & 163 & 181.5468 & 195\end{array}$

c)
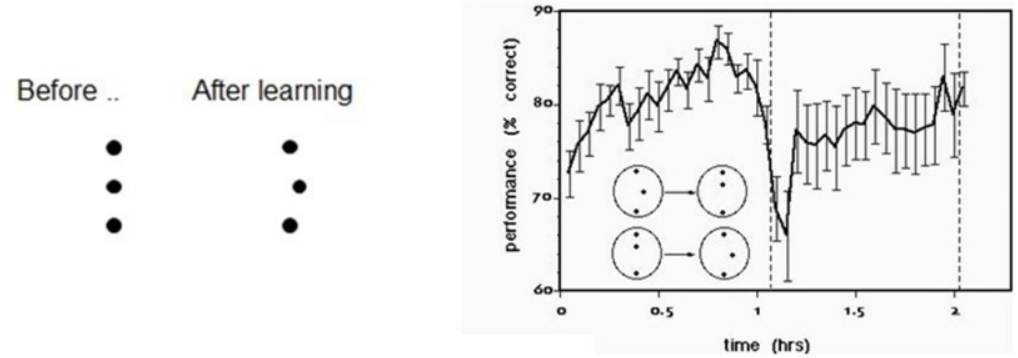

d)

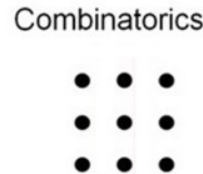

Figure 2. a) The Thatcher illusion: In the left image, it is hard to see that Margaret Thatcher's eyes and mouth have been flipped upside-down. The difference is immediately evident on the right with upright faces, illustrating the holistic nature of representations (Thompson, 1980). b) An image of Kant is shown on the left split up in sixteen squares each containing $12 \times 12$ pixels. On the right, we show the corresponding luminance values of one of the 16 squares. Which one? It is impossible to guess the correct square even though the information in Kant's picture and the numbers on the right are equivalent. The brain can extract information only in certain formats. There is a double dissociation. On the right, we see clearly that odd columns have post-comma values, even columns have no post-comma values. The corresponding luminance patterns is invisible on the left even though both representations are equivalent. c) Left. Perceptual learning in a dot bisection task: Participants are trained to tell whether a 
central dot is offset slightly to either the left or right of two flanking dots. Before training, the offset is largely invisible. The 3 dots seem to be aligned. With training, performance improves and participants can perceive small offsets. Right. One group of observers trained with a Vernier task: is the central dot offset either to the left or right (a right offset is shown)? The other group trained with a bisection task: is the central dot offset towards the upper or lower dot (an upper dot is shown)? Performance improved in both groups (results are collapsed for both groups). After an hour, the tasks were switched. No transfer of learning occurred, i.e., training with the vernier task did not improve performance in the bisection task and vice versa. Observers needed to learn again. The $x$-axis corresponds to the timeline of the training, the $y$-axis indicates performance, and the dashed vertical line represents the switches of the tasks. From Fahle \& Morgan (1996). d) Combinatorics: In a $3 \times 3$ dots configuration, there are 84 tasks with three dots that can potentially be learnt since any three dots can be chosen for the task. In general, an $n$-dot display can host $2 n$ tasks. For example, if $n=89$, there are more potential tasks than nanoseconds since the big bang. Importantly, learning one dot configuration does not transfer to other configurations.

Hence, things are not perceived simply because they are there: no representation, no perception. Here is one more example. Often it is believed that feelings of hunger and thirst just come by nature. They are simply there. However, this is not true. Nature came up with a complicated neural machinery to make us suffer when calories or water supplies are low. When the neurons coding for hunger die, which may happen for older people, there is no feeling of hunger anymore. People need to remind themselves to eat.

Conclusion. Since there is no 1-1 correspondence between objects and mental representations, the visual system needs to extract objects from the photoreceptor level. Perceptual systems can only perceive objects for which representations exist. No representation, no object recognition.

\section{Objects Ila: Learning}

Obviously, object representations need to come from somewhere. Representations may have appeared during evolution, been adopted during critical childhood phases, or learned during adulthood. For example, infants can compute depth visual information (Campos et al., 1978; Gibson \& Walk, 1960) and recognize faces (Field et al., 1984; Frank et al., 2009), indicating that these skills are innate. Conversely, kittens raised in an environment where horizontal elements are missing, are unable to perceive horizontal lines in adult life because the corresponding mental representations were not developed through stimulus exposure (Blakemore \& Cooper, 1970). Because of learning, radiologists spot a tumour on an X-ray scan with ease, which is impossible for novices. Sommeliers can recognize the grape of a wine, which is impossible for lay(wo)men. In both cases, long term perceptual learning has led to building up of corresponding representations which allow fine grained discriminations. Different types of ontogenetic and perceptual learning or loss of representations lead to changes in the perceptual system.

Conclusion. No representations without phylogenetic, ontogenetic, or adult perceptual learning.

\section{Objects IIb: Combinatorics}

Object recognition depends on learned representations. No learning, no perception. Interestingly, perceptual learning is usually very specific. Training to discriminate types of wine 
does not improve discrimination of tumours. The specificity of learning is even finer grained (Figure 2c). Observers are trained with a so-called dot vernier stimulus, i.e., two outer dots are bisected by a central dot, which can be offset either a bit to the left or right. Performance improves perception within an hour of training. Surprisingly, this improvement does not transfer to the same stimulus when the central dot is offset either a bit towards the upper or lower outer dot (dot bisection stimulus).

Only a tiny fraction of things can be learned. Consider the stimulus in figure $2 \mathrm{~d}$ with nine dots on a regular $3 \times 3$ grid, which may slightly be offset in whatever direction. $\frac{9 !}{3 !(9-3) !}=84$ tasks with three dots can be defined. Performance improves through training only for the learned task because there is no transfer. Tasks can also be defined with more than three dots. The number of combinations of $\mathrm{n}$ dots is $2^{n}$. Thus, with 9 dots, 512 tasks can be defined, for which there is no transfer from one to the other task. With 89 dots, there are more tasks than nanoseconds in the Universe. 89 is a very small number and there are not only dots in the universe but also lines, squares, and faces. Hence, there are zillions of potential tasks and objects, which can be learned.

Conclusion: Not all aspects of the world can be learned and hence represented. In fact, only a tiny, vanishing fraction can be represented.

\section{Objects Ilc: Subjectivity and individuality}

Humans can learn and thus perceive only a tiny fraction of all possible objects (figure $2 c \& d$ ). Since not all stimulus configurations can be learned, a selection is needed to decide what should be represented. In fact, it was shown that usually only attended aspects of a stimulus are learned (Ahissar \& Hochstein, 1993). This selection can be either self-guided or depends on instructions of others. Because there can be zillions of selections, there are potentially zillions of different perceptual systems. Hence, perception constitutes itself through perceptual learning (Herzog \& Esfeld, 2009). Cultural learning plays an important role. For example, radiologists learn as a community to perceive the world differently than other cultures, such as sommeliers. Likewise, people on different parts of the planet perceive illusions differently (Muller-Lyer illusion: Segall, Campbell, \& Herskovits, 1963, but see Todorovic, 2016; Ebbinghaus illusion: Caparos et al., 2012; de Fockert, Davidoff, Fagot, Parron, \& Goldstein, 2007; Ponzo Illusion: Leibowitz \& Pick 1972; see also Henrich, Heine, \& Norenzayan, 2010). Even within a population, perception of basic features (Bosten \& Mollon, 2010; Cappe et al., 2014) and illusions is very specific (Grzeczkowski et al., 2017). Appearance also strongly differs between humans in the example of \#thedress. Slightly more than half of the people perceive a picture of a dress as white and gold and the other part of the population as black and blue (Daoudi et al., 2017; Lafer-Sousa et al., 2015).

One might argue that radiologists perceive the world just like other people but, in addition, have some extra visual abilities. However, perception can also be truly different between observers. For example, assume that in the above example of the 3-dot stimulus, the central dot is slightly offset in both the horizontal and vertical direction leading to four alternatives: left-up offset, leftdown offset, right-up, and right-down offset. Before training, observers do not perceive any offset because the physical difference is small. One observer trains to discriminate the horizontal offset direction (vernier task), the other observer the vertical one (bisection task). Training 
improves perception specifically. After training, perception has improved but observers will now have different percepts and cannot agree on the offset direction (one observer perceives a horizontal but no vertical offset, and the opposite for the other observer). Even though perception has "increased", agreed truth has decreased.

Conclusion: Not all aspects of the world can be learned and hence represented. Different perceptual systems occur because of different learnings. Perception is individual and, hence, subjective.

\section{Metrics I: Perception}

Up to now, we have considered the mapping between single world elements, such as objects and faces, and the corresponding mental representations. On top of this, mental representations relate to each other. For example, we have the notion of distance, which relates elements to each other on a length scale. We also compare things relative to their weights, size, shape, beauty etc., i.e., there are many metrics. For a given comparison there are many possible metrics. For example, the subjective colour space is more or less circular whereas wavelength is linear (figure 3). Hence, in this case, the metrics are even topologically different. Likewise, there is no unique metric for distance. There are infinitely many, such as the Euclidean, Minkowskian, and Riemannian metrics. Depending on the metric chosen, conclusions can be quite different.

Conclusion: On top of having different representations, there can be many different metrics linking these representations, leading to different percepts.
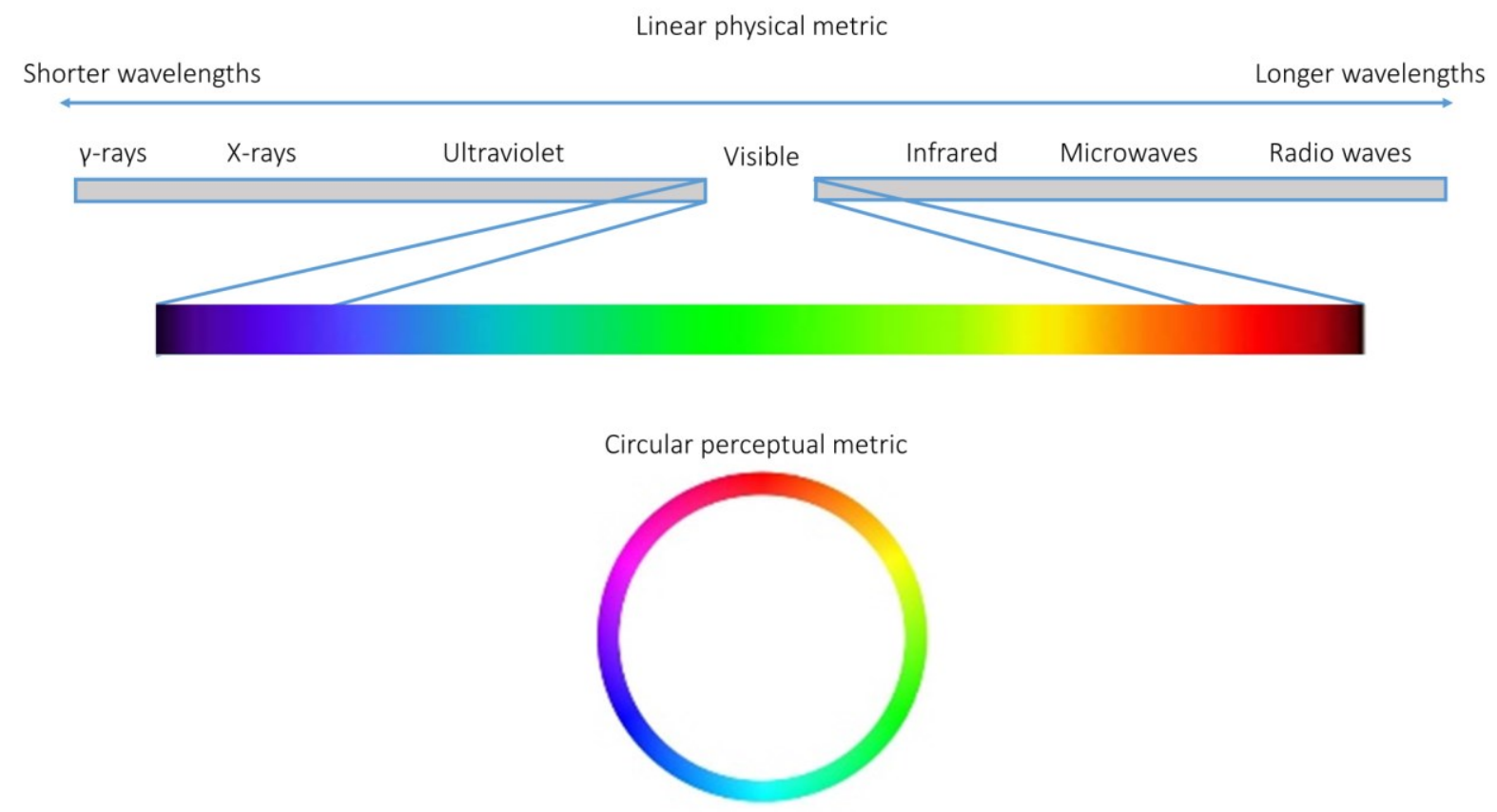

Figure 3: The physical space and the perceptual space are (topologically) different. The metric of the physical light is linear, ranging from $380 \mathrm{~nm}$ to $720 \mathrm{~nm}$. At a first approximation, the perceptual colour space is circular, i.e., neighbouring colours do not always correspond to neighbouring wavelengths. 


\section{Metrics II: Nested metrics and reference frames}

There are many metrics for many aspects, such as distance or colour, and for each aspect there are different instantiations. Hence, even in cases where object representations are identical, perceptual systems may strongly differ because their metrics differ. The overall metric of a perceptual system contains all instantiations of these metrics, e.g., a specific colour metric, a specific distance metric, and so on. In the simplest case, the metrics are independent. In this case, the number of all overall metrics is the multiplication of the number of the independent metrics. For example, one perceptual system may use a linear colour metric and the Riemannian distance metric.

However, the situation is usually more complex since metrics are nested in complex hierarchies, i.e., they are not independent. For example, metrics often depend on reference frames, such as in the example of the reflector on the bike wheel in figure 1. We may perceive the movement of someone's fingers relative to their waving hand, to their forearm, to their body, to the train in which they are leaving the train station and so on. Different perceptual systems may organize the perceptual space very differently- even if the number of object representations is identical.

Conclusions. Perception is not only individual because we represent different objects, it is also individual because we can represent the external world by different perceptual metrics. Even when representations are identical, perceptual systems may differ because of different incompatible metrics.

\section{Part II: The ontological status of objects}

In part I, we have shown that uncontroversial facts of vision science challenge the truth conditions of realism, i.e., it is questionable to what extent we can perceive objects veridically. However, these results do not question the existence of mind independent objects per se. Objects may exist, but we may simply not perceive them veridically because of the limited number of photorectors, ill-posed problems, reference frame dependency, missing representations, mind dependent metrics, etc.

In addition, we have shown that only a tiny fraction of things can be learnt and therefore perceived. Hence, perception is a many-to-one mapping which, as we show now, challenges the ontological status of objects as mind independent entities. In addition, we will show that realism is, for principled reasons, not the goal of perception.

Terminology: A mapping from a set $X$ to a set $Y$ is injective when for each $y$ in $Y$, there is at most one $x$ in $X$ with $f(x)=y$, i.e., $f\left(x_{1}\right)=f\left(x_{2}\right)$ implies $x_{1}=x_{2}$. Importantly, a non-injective mapping cannot be inverted because one cannot know which $x$ corresponds to $y$ (since there are many possible $x s$; figure 4c). Surjectivity of a mapping $f$ means that for each $y$ in $Y$, there is at least one $x$ in $X$ with $f(x)=y$. A mapping is bijective when it is both injective and surjective. For a bijection, also called a 1-1 mapping, each element in $X$ corresponds uniquely to an element in $Y$ (figure 4a). A bijection is invertable. An isomorphism is a bijective mapping, which preserves the metric of $X$. 


\section{Concepts I: Injectivity}

\section{Concepts la: Physics \& the number of objects}

Let us conceptualize the previous thoughts. Physics tell us that the world at a certain time can be described by a set of numbers, and the evolution of this set of numbers is given by equations. For example, according to the standard model, the world is composed of fundamental quantum fields whose state is described by quantum numbers. These states can be summarized by a vector $w(t)=(w 1(t), w 2(t), \ldots, w N(t))$. For example, this world vector can describe the state of the universe one second after the big bang or at this very moment (figure $4 d^{1}$ ). The evolution of this vector is dictated by a Lagrangian, giving rise to differential equations that describe how the fields change. Importantly, our arguments do not depend on the veracity of the standard model. They only require that the world can be described by a list of numbers (as is also the case, for example, in Newtonian mechanics or string theory).

World states can be split up into internal states of a perceiver and external states. Sensation can be seen as a mapping $f$ from the world vector onto the retina (or other sensors). Each world state produces a specific pattern on the retina. Perception is a further mapping $g$ from the retina to the set of representations of the perceptual system.

There are many more external than internal states. First, only a tiny subset of the entries of the world vector projects onto the retina at a moment of time. For example, objects in the back of the head do not activate the retina. Even within the visual field, states may not activate the retina if they reflect light of invisible wavelengths, and, for visible wavelengths, many states are indistinguishable because of metamers (see Part I: Sensation). Hence, there are many world states projecting onto the same retinal pattern. Hence, the world-retina mapping $f$ is not injective and, thus, cannot be inverted (figure 4c). We cannot infer which of the many potential world states gave rise to the current retinal pattern.

Second, with only the photoreceptor sheet, we cannot perceive any objects (see Part I: Objects). We need to extract objects, which means we need to map retinal images onto representations (figure 4d). There are many retinal patterns mapping onto the same representation. Hence, the mapping $g$ is not injective either and therefore cannot be inverted. For example, assume a photoreceptor can only be active or inactive (which is an oversimplification, because photoreceptor activity covers a wide range of values) and there are $n$ photoreceptors. Then, the number of retinal patterns is not $n$ but $2^{\text {n }}$, i.e., the power set. The human retina of one eye has more than 100 million photoreceptors, hence, there are $2^{100.000 .000}$ patterns. The number of nanoseconds in the universe is much smaller, namely $2^{89}$. The number of world states $m$ is even larger and the number of all potential objects is not $m$ but $2^{m}$, i.e., far beyond what can be coded in a brain. Obviously, a perceptual system can represent only a tiny fraction of all world states, so the mapping from world states to object representation is not injective and cannot be inverted.

\footnotetext{
${ }^{1}$ Mathematically speaking, the notion of a vector is slightly misleading because the world "vector" as we use the term is simply a list of states, without the requirement to live in a vector space. However, we keep the term "world vector" for simplicity.
} 
As a side comment, one might argue that some retinal patterns may never occur, if there are no world states that give rise to these patterns. However, we can easily create visual patterns on computer screens, which create all types of retinal patterns.

Conclusion. Sensation and perception are both many-to-one mappings. There are simply too many potential objects to be represented. Since perception is not injective, perception cannot be inverted, i.e., when $y$ is perceived one cannot know which $x$ caused $y$.
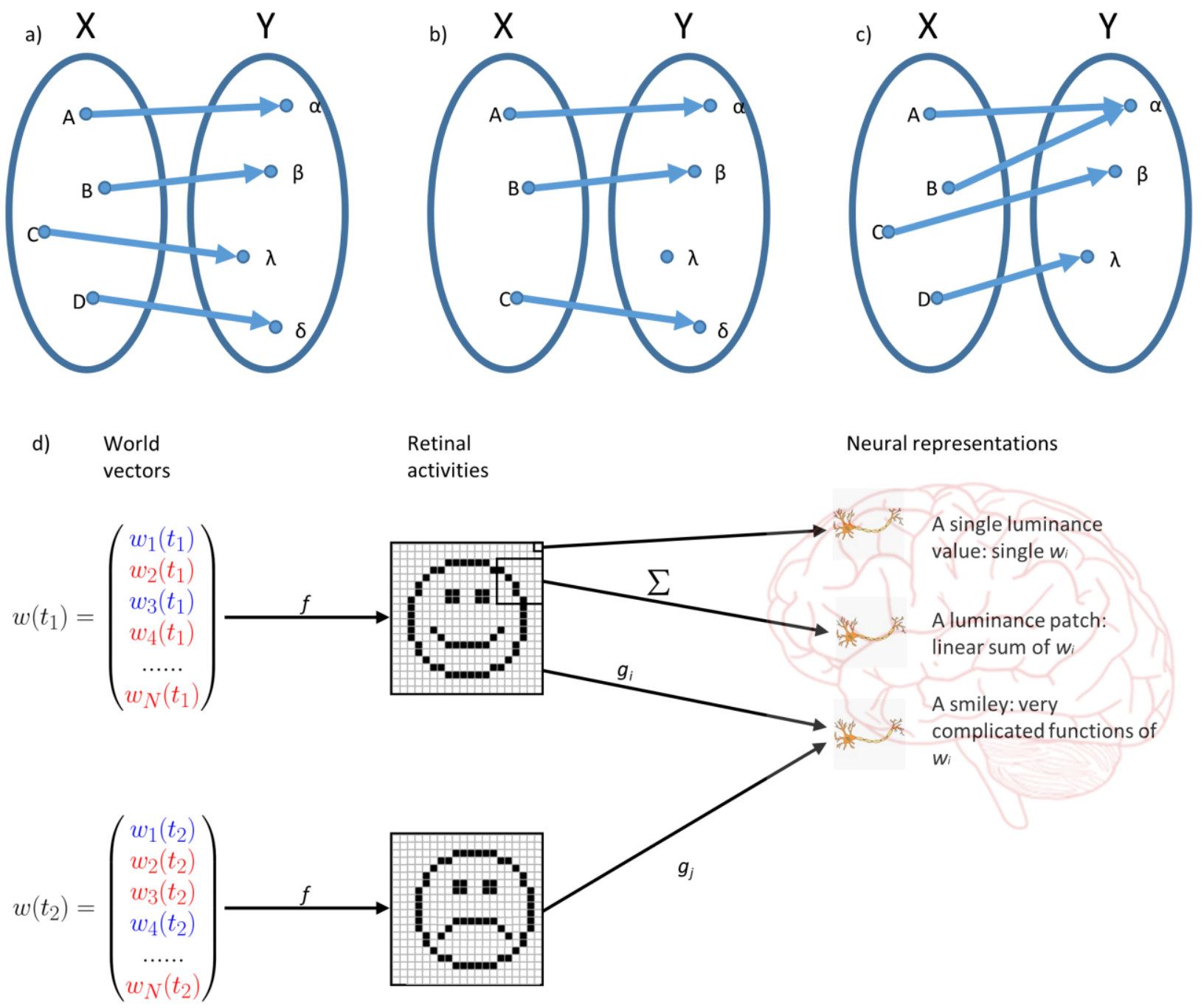

Figure 4. a-c) Mappings. a) A mapping is called bijective or 1-1, when for each element in the set $X$ there is exactly one element in the set $Y$ and vice versa. Realism assumes that perception is bijective, i.e., there is 1-1 correspondence between objects in the external world and mental representations. A bijective 
mapping is invertable, i.e., one can find for all elements $y$ in $Y$, the unique element $x$ in $X$ that maps onto $y$. Perception is not bijective because there are more external world states than representations. Hence, perception is not invertable. We can never find out which world states give rise to a percept. b) Example of an injective function, which is not surjective. c) Example of a surjective function, which is not injective. A function is bijective if and only if it is injective and surjective. d) World vector, mappings, and representations. The state of the world at time $t$ is described by a world vector expressing the current state of the fundamental entities of the world (e.g., the quantum numbers of quantum fields). Some entries of $w$ impact on a perceptual system (blue), whereas others do not (red). The colours of the entries change over time. Other entries (not shown) correspond to states of the perceptual system. Sensation can be seen as a mapping $f$ from the world vector onto the retina (which itself is part of the world vector). If the retina has $n$ neurons, which can only be active or inactive (i.e., 1 or 0 ), there are $2^{n}$ possible retinal activities. In the toy example of the smiley, there are 400 pixels, so $2^{400}$ possible retinal activations, much more than the number of nanoseconds since big bang. Perception can be seen as a mapping $g$ from the retina onto object representations or as the composite mapping of $f$ and $g$. In the so-called grandmother coding theory, a single neuron or a few neurons respond to a certain object (Barlow, 1972). For example, when grandmother enters the room, a grandmother neuron fires indicating the presence of grandmother. Likewise, we can detect a smiley because we have neurons coding for the smiley. Another neuron may code for the overall luminance of a patch of retinal image or the entire retinal image. In this case, the mapping is as simple as summing up all luminance values. All these representations come from the very same retinal image. Small changes on the retina may lead to large differences in the perceived emotion of the smiley. Smileys with different emotions are still perceived as the same smiley because there are neurons coding for the smiley independent of the emotion. Even though all information is on the photoreceptor level, there is no object recognition on the retina. If there is no mapping from the photoreceptors to a mental representation, there is no perception (Part I: Objects).

\section{Concepts Ib: Partitions \& the doubling of ontology}

Many physical states are mapped onto one retinal pattern and, subsequently, onto a mental representation. We can see a mental representation as "representative" for the physical states leading to that representation. Hence, a representation partitions the world into subclasses of states. A subclass contains all the states, which project on one "object representation". Partitions are not unique because of the many-to-one mapping. Hence, there is no unique way to represent the external world.

Here is a toy example. Assume the world vector has only 4 entries represented by 4 numbers, which are either 0 or 1 . Hence, there are $2^{4}=16$ states. Assume there are two perceptual systems that can sense the full world vector through 4 photoreceptors, and each of these systems has only 2 object representations. For example, perceptual system 1 may map the 8 states with more than 2 zeros on object 1 and the others to object 2 . In the perceptual system 2 , only $(0,0,0,0)$ and $(1,1,1,1)$ are mapped onto object 1 and the other states are mapped onto object 2 . In total, we

can create $2^{16-1}$ - 1 perceptual systems all perceiving the mini-world differently, i.e., having their own partitions (the exact number depends on what counts as different systems). Hence, there is no unique way to represent the external world. Even though the world states are mind independent, the perceived objects depend on the perceptual system. Objects are therefore subjective and mind dependent. Realism would only be true if the 16 states were mapped 
bijectively onto 16 representations. In this case, realism would be nothing else than a look up table, similar to a telephone book ${ }^{2}$.

We would like to reiterate that systems cannot infer the world states from their percepts. In our example, system 2 cannot tell whether it perceives object 1 because of $(0,0,0,0)$ or $(1,1,1,1)$. There cannot be a shared ground truth for these 2-object systems. Both systems map $(0,0,0,0)$ onto object 1 and hence the percepts match. However, for $(0,0,0,1)$ there is no "agreement". In fact, the percepts are the same in half of the cases and different in the other half, i.e., they are fully uncorrelated.

These considerations are even more pronounced in complex systems. For instance, deep neural networks may classify certain images like humans but perform completely differently with other cases (as in adversarial examples; llyas et al., 2019; Jacobsen, Behrmann, Zemel, \& Bethge, 2018; Su, Vargas, \& Sakurai, 2019; Szegedy et al., 2013). In general, it can be shown mathematically that any possible perceptual system can be constructed with a single hidden layer network, i.e., there is always a network that realizes any arbitrary world-mind mapping or partition (Hornik et al., 1989). In the animal kingdom, different partitions are driven by different ecological needs so there are similarities between individuals of similar species, but also differences, as the arguments in part 1 show.

Here is a non-perceptual example of a non-injective, and therefore non-invertible mapping, and different possible partitions. The Olympic committee, located in Lausanne, evaluates the success of a nation at the Olympic games by a simple algorithm: the more gold medals, the higher the rank. This measure is a non-injective mapping. It collapses rich information into one number and there are other ways to do so. For example, one may also take the silver and bronze medals into account. One might weight them ( 3 for gold, 2 for silver, 1 for bronze). One may normalize by the size of the population. There are many ways, and there is not one "more veridical" way than the others.

The fact that there is a large number of possible partitions leads to a serious problem for realism. Realism assumes the existence of a mind independent world of objects, in addition to the physical states. However, this would double ontology in an unhealthy, non-unique way (see also Merricks, 2003). On top of the observer-independent physical states, there would be an observerdependent world of objects "in between" the physical world and the mental representations of the perceiver (figure 5). Worse, these objects would depend on idiosyncrasies of the observer's perceptual system (figure 4). Hence, perception is not a mapping from the world vector to the set of objects followed by a mapping from the set of objects to mental representations. Perception is a direct mapping from the world vector to mental representations (via sensation). Objects are identical to mental representations.

\footnotetext{
2 Even in this case there is no unique representation, in a certain sense. For example, $(0,0,0,0)$ may be mapped on object 2 in system 1 and on object 1 in system 2. However, it may be debated whether these are truly different systems.
} 


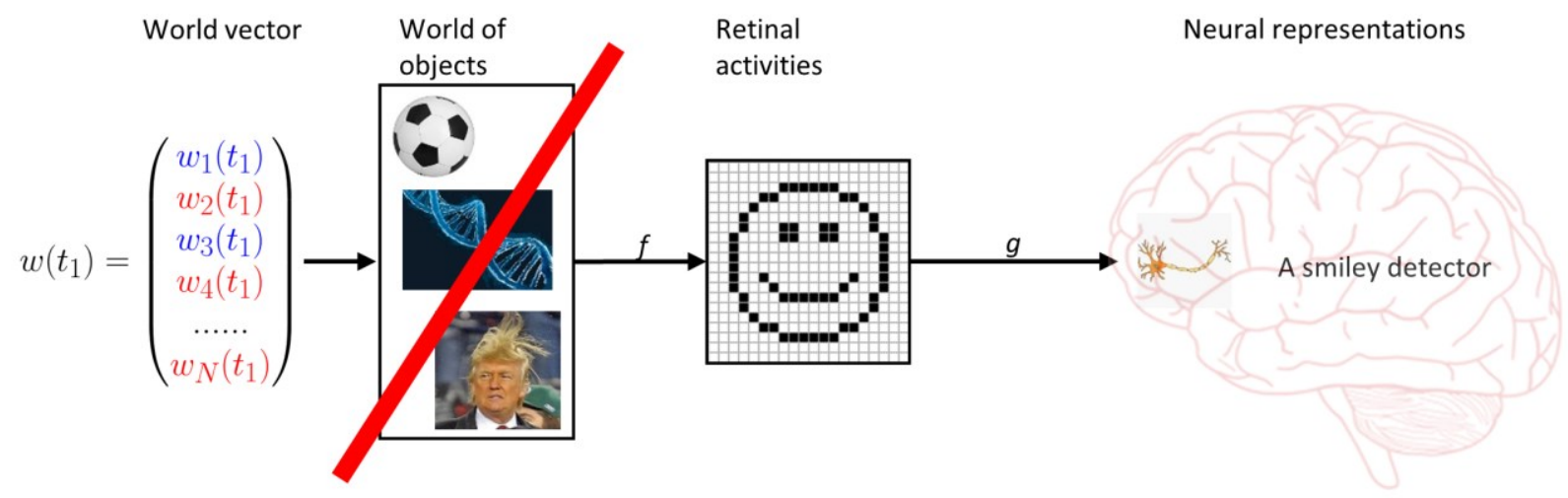

Figure 5: Doubling of ontology. Perception can be seen as a mapping from the world vector to percepts. Positing an intermediate "world of objects" unparsimoniously doubles the ontology. Worse still, the "world of objects may be different for each perceptual system.

We identify objects with the external world states because of their seeming causal power and independence, and because we are not aware of the many states giving rise to the same representation, i.e., we do not notice the non-injectivity. However, objects are the outputs of the visual system and not its inputs (Mausfeld, 2015).

But aren't objects composed of atoms, molecules, etc., and, therefore, exist in a mindindependent fashion? No. Only the world vector exists in a mind-independent fashion. Atoms and molecules are mind-dependent constructions, and so are all the other entities of our physical theories, including light, heat, etc. Only the fundamental entities described by the world vector are mind independent.

In the toy example above, the world comprised all possible vectors (i.e., $\{(0,0,0,0),(0,0,0,1)$, $(0,0,1,0),(0,1,0,0),(1,0,0,0),(1,0,0,1),(1,0,1,0),(1,1,0,0),(0,1,0,1),(0,1,1,0),(0,0,1,1),(0,1,1,1)$, $(1,0,1,1),(1,1,0,1),(1,1,1,0),(1,1,1,1)\})^{3}$. In the real word governed by the physical laws, many states may never occur. This means that not everything is possible to perceive. Still, the number of world states is much larger than the number of mental representations and hence there are many ways to perceive the world.

Conclusion. Ontologically, the world contains only the fundamental entities described by the world vector. Perception is a direct mapping from these fundamental states to mental representations. The idea of a mind-independent world of objects doubles ontology in an unhealthy, observer dependent manner. Objects are identical with the mental representations.

${ }^{3}$ In this example, we have assumed that mental representations split up the world in disjunctive classes. However, this is not necessary for our argument. 


\section{Concepts Ic: Invariants \& the goal of perception}

Up to now, we have focused on the many indistinguishable states, which all map onto one representation. Which of these many states elicits the representation at a certain moment is unknown because we cannot invert the mapping. This non-invertibility continues with distinguishable states perceived as the same object. For example, when grandma changes her facial expression, we still perceive her as the same person, and not as someone else. There is an "invariant" representation for grandma, which "bundles" many different percepts of grandma. These "bundled", invariant representations are crucial for learning to act. For example, one might get candy no matter what hat grandma wears. Likewise, turning an object by only a few degrees leads to a highly different retinal image and to different percepts. Still, we perceive the same object- not because it is "veridically" the same object, but because we map the corresponding states onto the same representation. Other perceptual systems may do so differently.

In this respect, one may say that the representation of grandmother is equivalent to all potential instantiations of grandmother's face (all views, all emotional expressions, all possible clothes). However, there is no grandmother in the world vector. Grandma is created by lumping many world states onto one representation. It is the partition that matters.

As mentioned, how we create invariants is not arbitrary but depends on goals. Many states come with similar consequences. Grandma states are related with candy states. Tiger states are related to states where you are a tiger's candy. Hence, the invariance is not in the external world states, it is in the consequences for other observer-dependant aspects. Percepts are not about predictions of the external world but about predictions of other perceptual outcomes, which are nothing else than other invariances.

Hence, the goal of perception is not to create a 1-1 image of the external world. The goal of perception is to lose irrelevant information by mapping many external world states onto one representation that is useful for survival.

Importantly, the notion of invariance does not imply that all perceptual systems perceive the same invariants. Quite to the contrary, what is invariant in one system may not be invariant in another system.

An invariant is the ability to not discriminate between states. There is a trade-off. The smaller the number of invariants, the compacter and simpler the representation of the world - at the cost of missing distinctions. It is a mathematical fact, captured by the so called no-free-lunch and ugly duckling theorems, that optimizing a certain perceptual process in neural networks comes at the cost of other perceptual functions (Wolpert \& Macready, 1997; https://en.wikipedia.org /wiki/No free lunch theorem; https://en.wikipedia.org/wiki/Ugly duckling theorem).

As a first side comment, object representations may be seen as abstract representations. For example, the mean value of throwing dice is 3.5 , but this value is never encountered for any particular throw. Similarly, a person is constantly changing; still we maintain a notion of invariance of the person, which does not need to correspond to a particular instance of that person or a pictorial average of the retinal images elicited by that person. Indeed, what would be the "veridical" or most representative view of grandma?

As a second side comment, objects can be seen as a similarity measure. Views that lead to the perception of an object are closer to each other than views that belong to different objects 
according the observer's perceptual metric. As explained earlier, there are many ways to do so (see Metrics I \& II). For this reason, similarity is always subjective. Similarity is in the eye of the beholder.

As a third side comment, there is no veridical, mind-independent object identity. Object identity is the same as object invariance.

As a fourth side comment, there are not only invariant representations for individual objects or persons. There are also representations for classes such as horses vs. cows. Whereas the difference between object instances and object classes is conceptually important, the principle of extracting invariants is the same: it is a non-injective mapping.

Conclusion: The goal of perception is to create invariants, which precludes object realism and 11 correspondences since there are many ways of creating invariants. Realism is not the goal of perception. Losing information is not only inevitable but actually good, at least to a certain extent. How to lose information is not unique. Different ways of losing information define different perceptual systems.

\section{Concepts Id: Structure \& metrics}

There is no structure in the world vector. As with objects, structure can be extracted in a mind dependent matter from the vector, which is reflected by the fact that, as mentioned in Section Metrics I \& II, there are many metrics. We impose metrics on the world states by putting representations in relation with each other.

Conclusion: Metrics, and hence structure, between mental representations (i.e., between objects) are in the mind of the perceiver. Therefore, structural realism (Ladyman, 2014) faces the same problem as any other type of realism.

\section{Concepts le: Some words on terminology}

Importantly, the notion of re-presentation is misleading because there are no objects in the external world and, hence, there is nothing to re-present. We still keep the term for traditional reasons. In addition, we use phrases such as "we can extract only a small fraction of potential objects" even though strictly speaking we cannot extract objects from the external world because objects are in the mind and not in the external world. We could eliminate the notion of objects and use only the term mental representation. The same is true for the term "invariance" because "invariants" are created by a metric, and are invariant only relative to this metric.

\section{Concepts II: (Non)-surjectivity}

Philosophers have worried about the non-surjectivity of perception for millennia, i.e., about things we perceive but which are not out there, such as hallucinations and unicorns. On several occasions, the seemingly non-surjectivity of perception has prompted philosophers to abandon the external world leading to idealism or solipsism. However, non-surjectivity is only a problem if one believes that perception is or needs to be realistic, i.e., that there is a 1-1 mapping between the external world and mental representations. 
First, the percepts in hallucinations "are what they are", i.e., they are just as proper percepts as non-illusory cases. One may feel deceived because there is no external world cause and decide to make a distinction between objects which occur only in drawings, fantasy, etc. and which occur in nature. However, this is a cognitive and not a perceptual issue. In this sense, hallucinations tell us that not only external states but also internal states can activate mental representations. In fact, mental representations can directly be activated by stimulating the corresponding neurons (Kammer et al., 2005). Hence, non-surjectivity is only surprising when one assumes that perception is triggered exclusively by external world objects and that there is a 1-1 mappingwhich is obviously not the case as we know from dreams and mental imagery. Bistable stimuli, such as Wittgenstein's famous duck-rabbit, are another example in which not only the external world, but also "internal" brain processes influence perception.

Second, we can imagine an elephant. It is therefore not surprising that we can also imagine a unicorn. Both come with abstract representations and do not "picture" anything real, just as the mean value of 3.5 never occurs when throwing a die. In this sense, hallucinations may be seen as uncontrolled mental imagery.

Whereas all these considerations are relevant for the philosophy of perception and in particular for the question of what we perceive when we perceive objects, these considerations are not crucial for the ontological questions about objects, which are of primary interest here. In some way, non-injectivity is about the ontology of objects, i.e., whether or not say exist. Nonsurjectivity is about the nature of mental representations, i.e., how they are generated in the brain.

As a side comment. Perception is sometimes called controlled hallucination (Koenderink \& van Doorn, 2008; Seth, 2021) or delusional (Lotto, 2017). We would rather say that hallucinations are caused by internal, rather than external, sources and that perception is not a delusion because delusions presuppose ground truth.

Conclusion. Non-injectivity, not non-surjectivity, is the main problem for realism.

\section{Concepts III: Self-consistency}

We have shown that "objects" are subjective and may differ between different perceptual systems. However, our argumentation in part I strongly depended on terms such as objects, light, and reflectance. Hence, there seems to be a contradiction. Perception science seems to rely on things, which do not exist. This is, in fact, true for the epistemological aspects outlaid in part I, but not for the ontological argument of part II. In part II, we only presupposed that monism and particle physics are true in the sense that all world states can be uniquely expressed as a vector ${ }^{4}$. Because of monism, perception must be a mapping from this vector onto a subset of it (we do not even need the notions of sensory systems, brains, etc.). Physics tells us that there are more world states than representations and, hence, the mapping is not injective and, for this reason, perception is not unique. There can be many partitions. Hence, this analysis does not rely on specific terms but only on the notion of mathematical mappings. Creatures with different

\footnotetext{
${ }^{4}$ We presuppose only the existence of the fundamental physical entities described by the world vector, not of other physical entities such as molecules, heat, pressure, stars, etc.
} 
perceptual systems would arrive at the same conclusions. In this respect, the above analysis is self-consistent.

In part I, perception theory partitions the world into certain classes, similarly to how perception partitions the world, but on a cognitive level. Indeed, there might be other partitions, which correspond to other perception theories with different entities and different conclusions. The theory of perception in the far future may look quite different than our current one- the wellknown pessimistic induction argument (Laudan, 1981). However, it is questionable whether any theory of perception of the near-future will deliver a fundamentally different picture making the above considerations obsolete.

Conclusion. The ontological arguments of this contribution are self-consistent. Hence, for the moment, our best theories of physics and perception do not provide any evidence for realism about objects, neither epistemologically nor ontologically. Thus, we must either give up physicalism or realism.

\section{Discussion}

For more than 2000 years, the relationship between the entities of the outer world and their mental counterparts has been an endless discussion. It has been doubted whether truth can be based on perception because the senses are limited (Plato, 375BC/2000), and because perception is perturbed in hallucinations (Descartes, 1637/1996), illusions (Hume, 1739/2003) and ambiguous figures (Wittgenstein, 1953/2009). For this reason, idealism entirely abandoned the idea of an outer world. The problems with idealism are well known and are not considered here.

In contrast, for empiricists, it is just the other way around: basic percepts provide ground truth (Barnes, Bloor, \& Henry, 1996; Popper, 1959/2005; Reid, 1764). Direct realism proposes that objects are directly perceived, e.g., sensation itself provides a veridical "picture" of the world. Indirect realism proposes that we perceive objects only indirectly, for example via sense data. Neuroscience and perception science usually (implicitly) subscribe to indirect realism: sensation does not reflect the "true" properties of objects but later stages do. Both direct and indirect realism presuppose an isomorphism between the elements of the external world and the mental representations. Because of this isomorphism, Occam's razor suggests that subjectivity can be replaced by objective neural descriptions, a position advocated by philosophical eliminativism (Churchland \& Churchland, 1998).

Here, we have shown that both direct and indirect realism about objects are not tenable positions. In fact, physicalism leads to anti-realism about objects. Because there is no isomorphism between percepts and the world, perception is essentially subjective. Importantly, this anti-realism is not a form of idealism since we presuppose a mind independent world, which is well described by particle physics. In a forthcoming contribution, we will argue that similar arguments apply to scientific realism as well.

In the following, we consider potential objections to our anti-realist argument and contrast our position with other positions. A discussion of how our proposal relates to classic philosophical positions is given in the appendix. 
Truth conditions. In Part I, we have shown that even if there were a mind independent world of objects, we could not perceive these objects veridically because of metamers, non-unique reference frames, and many other aspects.

Direct realism is still popular in the philosophy of science (e.g., Van Fraassen, 1980). Nevertheless, direct realism can be ruled out right away because perception is ill-posed and hence the photoreceptor level tells us the least about the world. The brain needs to construct a useful percept from sensory data by relying on subjective invariants. As a side comment, if sensation delivered ground truth, the question would arise why humans carry a large brain with them, containing about 40 visual areas eating up a third of the calories going to the neocortex. The retina is sufficient for direct realism. The reason for this large-scale brain investment is that the photoreceptor level is only the starting point of perception.

Indirect realism is precisely about solving the ill-posed problems of the photoreceptor level by taking contextual and prior information into account. However, there is no unique solution to these ill-posed problems, even using priors. First, priors that are useful in certain situations are useless in others, e.g., when the light source does not come from above (figure 1f). Often it is argued that evolution has found the optimal priors. However, evolution is not about delivering a veridical image of the world but rather a useful one (see below; Hoffman et al., 2013; Koenderink, 2014; Koenderink \& van Doorn, 2008; Lotto, 2017).

Structural realism proposes that realism is more about structures than entities (Ladyman, 2014). However, just as with reference frames, there are many metrics even for basics aspects such as distance, and hence there is no privileged one. For this reason, structures are as underconstrained as entities.

Schwartz (2016) adds an interesting philosophical argument to the above physiological ones. How could we veridically perceive that a building is $80 \mathrm{~m}$ high and not $81 \mathrm{~m}$ ? Whereas realism may work for the existence of objects, it fails for comparisons of object features because there is no proper reference frame.

Evolution. Often it is proposed that evolution leads to realistic perception because, otherwise, perceptual systems could not survive. However, this is not a viable road.

First, although evolution indeed excludes many systems, it neither leads to a unique perceptual solution nor to a realistic one (review: Hoffman et al., 2013; but see Mausfeld, 2015). We know that evolution has created many highly different perceptual systems. Moreover, different ecological needs lead to different partitions. It is sufficient to realize that a tiger is approaching without the explicitly coding for female vs. male. On the other hand, the female vs. male tiger distinction is ecologically relevant for tigers.

Second, although perception is made to interact with the environment, there are many ways to realize actions with similar outcomes. For example, although gravity is crucial for all species, different species cope with it in different ways.

Third, whether or not different perceptual systems actually exist is not crucial (although the arguments in Part I suggest that they do). For the above argumentation it is sufficient that they could logically exist, which is the case because we can construct arbitrary perceptual systems (Hornik et al., 1989). 
In general, it is one thing to trust perception in everyday life. It is another issue to claim that perception necessarily delivers a veridical image.

Inverse optics. Often, it is proposed that vision performs inverse optics, making inferences to solve the inverse problems of vision (Marr, 1982; von Helmholtz, 1883). However, we cannot invert vision because the world-percept mapping is not injective and therefore not invertable. The world is much richer than our percepts and the world comprises no objects - just states of quantum fields - which we cannot and do not want to perceive. The goal of perception is not to predict the outer world but to predict future percepts, for example, depending on other percepts or actions etc. (Hoffman et al., 2013; Luhmann, 1986; Mausfeld, 2015; O'Regan \& Noë, 2001; Putnam, 1981). Similar arguments apply to the Bayes approache (e.g., Vilares \& Kording, 2011), which assumes that there is a 1-1 mapping between outer world objects and percepts.

What does "solving" ill-posed problems mean? Material properties, e.g., the "true" reflectance of an "object", are in the outer world, which we do not perceive veridically. Thus, what is the visual system "really" doing when it solves the ill-posed problems of vision? It is extracting information that is predictive of future percepts. For example, to compute the colour of an object, knowledge of the illuminance is needed (Part I: Perception II). There is no fundamentally "privileged" illuminance, just like there is no privileged reference system for motion (Part I: Perception II). The human visual system uses the light of the sun as a reference illuminance because it is useful in our particular ecological niche. It transforms the current illumination as if there were sunlight shining on the object. For example, the brain knowns that, under sunlight, a red strawberry is tasty whereas a green one is not. By using colour constancy, knowledge about strawberry taste can be used under all illuminances, even ones not encountered previously. However, other perceptual systems can use other reference lighting conditions. For example, red light cannot reach the deep sea, so the reference conditions for creatures living there is different.

Complex percepts. Importantly, in this contribution, we talked about percepts in the most basic sense such as simple object detectors: the presentation of an object leads to the percept of this object in a causal manner. Human percepts go much beyond such object detectors. For example, mental representations are influenced by each other and not only by the external world, they include history, emotional aspects, etc. One might argue that this changes the situation. However, the same arguments hold for these more complex representations. Likewise, we did not differentiate between conscious and unconscious perception because all of the above considerations hold true independently of whether perception is conscious or unconscious.

Physical realization. One may argue that our arguments lead to dualism, idealism or solipsism, because of the mind-dependence of objects. However, whereas the objects are mind dependent, the physical states that give rise to these objects are not. In addition, these physical states are scientifically accessible and partially computable by the laws of particle physics. Moreover, sensation and perception (the $f$ and $g$ mappings described in Part II), are physical processes that occur by well-studied neural machinery. Finally, not everything can be perceived because the physical states impose strong constraints on what can be perceived at a given moment. However, the constraints are not strong enough to enforce a unique percept.

Interpretation. It is often proposed that there is ground truth in perception and at certain occasions the human brain "adds" subjective interpretations. Proposals strongly vary about the 
ratio of veridical perception and interpretation (Barron \& Klein, 2016; Frith, 2013; Grossberg, 2013; Hofstadter \& Sander, 2013; Lotto, 2017; Searle, 2015). For example, we perceive columns of dots even when there are only dots because we group the dots by the so-called Gestalt principle of proximity (figure 7). It is often proposed that the columns are subjective but the dots are objective (Kanizsa, 1979). In contrast, our argument proposes that perception is entirely subjective, i.e., both the dots and the columns are subjective, because they all depend on noninvertible mappings.

To put it another way, only the particles of physics are real. Everything else is interpretation. When we perceive a candle and we close the eyes, the candle disappears but the particles, that gave rise to the candle percept, stay, i.e., they exist whether or not we perceive them.

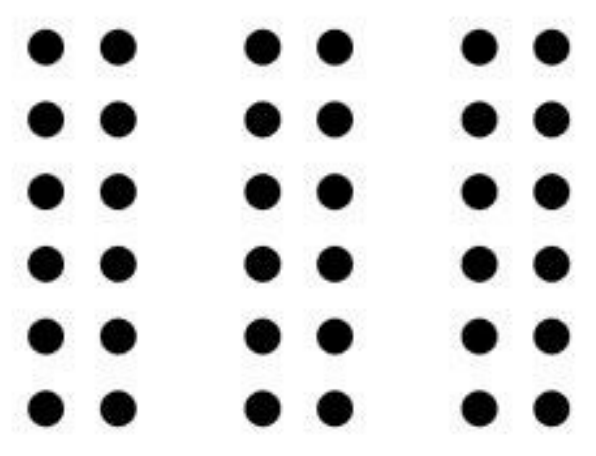

Figure 7. Gestalts. The dots are perceived as forming columns because individual dots are grouped by proximity.

The Goal of perception: tokens and types. Direct perception of a physical state, i.e., a token, makes little sense. It is perception of just this token without any meaning. If perception were about tokens, perception would be a stream of unrelated states. Two representations would have nothing in common, even if the represented features differ only slightly, say two faces that differ only by a small change in the opening of the mouth (figure 3a). In token realism there is no generalization. The goal of perceptual systems is just the opposite, stipulating a similarity metric on different instances of the photoreceptor level and classifying them as belonging to the same or a different object, scene, or background.

The goal of perception is to project the high dimensional world vector on a small perceptual space, which captures invariants relative to a set of internal metrics. For this reason, the goal of perception is to find non-injective mappings that "loose" information in a useful way. In fact, the mathematical equivalent of "loss" is a non-injective mapping.

For species that communicate with each other, similar perceptual systems are necessary. That is why expert radiologists train novices to perceive the world as they do, and this is the reason why perception seems to be so reliable and unequivocal. If humans had highly incompatible perceptual systems they would be unable to communicate.

The Super-perceiver. The facts presented in part I are not new and they are not controversial. It is surprising how little attention neuroscience, psychology, and computer vision have paid to the implications of these facts (with notable exceptions, see Hoffman, 2019; Koenderink, 2014; 
Koenderink \& van Doorn, 2008; Lotto, 2017; Mausfeld, 2015; Smith, 1996).Three reasons come to mind to explain why realist intuitions are still predominant.

a. Reliability. Perception seems to be largely reliable and mis-perceptions are usually rare and recognized as such. Humans rarely disagree whether or not an object is there. Individual perceptual differences are rather the exception than the rule. This could suggest that, even though there is no ground truth, there may be "approximate" truth. In fact, most neuroscientists view the brain as a machine that actually delivers a veridical image of the external world by, amongst other tricks, solving the ill-posed problems of vision.

b. Extension. Even though sensation is limited, for example restricted to the small range of visible light, perception delivers a realistic image of the external world within this range, i.e., bijective correspondence holds true within this range. In addition, the missing information can be retrieved using non-human sensors, such UV-sensitive cameras. Similarly, we cannot see the micro-structure of liver cells because the resolution of our eyes is limited. However, we can extend perception by using a microscope. Were it be possible to insert UV-sensitive receptors in the human retina, rainbows would be perceived with one additional color: ultraviolet next to blue. Missing information could in principle be filled in. We cannot perceive everything, but when we perceive something, we have a realistic image of this thing and 1-1 correspondence.

c. Existence. Inaccurate perception does in no way disprove the existence of a unique external world of objects, comprised of grandma and candy.

The Super-perceiver. In some way, these points underlying common realist intuitions imply the possible existence of a super-perceiver, who is sensitive to all physical information, creating a perfect realistic image of the external world. The perceptual systems of all creatures are subsets of the perceptual system of the super-perceiver.

However, there cannot be a super-perceiver. First, the super-perceiver suffers from reference dependence just as any other perceiver (Part I: Perception II). The super-perceiver would need to perceive the reflector on the wheel within Euclidean, object centered, etc. coordinates all at the same time (figure 1g). Second, the super-perceiver could not perceive all objects because there are too many. If the size of the world vector is $n$, the number of potential objects is not $n$ but $2 n$ because each object is a point in the power set of the world vector. Hence, the perceptual space is combinatorially larger than the world vector. Third, similarly to the first point, there is no unique perceptual metric, but many incompatible ones. For example, the human colour space is circular, but the colour space could be linear or shaped like an 8 for other perceptual systems. A super-perceiver would need to perceive blue in between red and green AND red between blue and green at the same time. Instead of one percept, there would be many conflicting percepts for the same world state. Perception can be fundamentally different for different perceptual systems and it is impossible to summarize them in a single super-perceiver.

There is only a single fundamental ontology. There are no mind independent objects but only fundamental physical states. If objects existed independently of the observer, this would inflate ontology because there is no unique relationship between objects and physical states. Objects refer to classes of internal physical states - they are identical with mental representations. They are types, not tokens. 
The above considerations hold true in many other cases. If one has a fundamental ontology, such as particle physics, there cannot be other ontologies, such as objects, as long as these are not bijective to the fundamental ontology. All non-fundamental entities are mind dependent.

\section{Summary}

Realism presupposes a bijection (or at least an injection) between the external and internal world. There is a 1-1 correspondence between an outer-world candle and its mental representation. Perception provides ground truth. Although realism about objects makes intuitive sense, it is often not quite clear what exactly is proposed to correspond to what. Whatever the specific interpretation is, we have shown that realism about objects cannot be true and is not the goal of perception. The main argument is that perception is always a mapping from a larger into a smaller set and is therefore not injective and invertible. There are always more world states than perceptual states and, hence, one can never know which world state is actually present. Because perception is not injective, there are always many potential ways to "represent" the world. Hence, perception cannot be unique, so realism of objects entails an unhealthy doubling of ontology. Realism about fundamental physics precludes realism about objects.

\section{Acknowledgements}

We would like to thank Richard Walker and Matteo Grasso for useful comments on the manuscript. Adrien Doerig was supported by the Swiss National Science Foundation grants n.176153 "Basics of visual processing: from elements to figures" and n.191718 "Towards machines that see like us: human eye movements for robust deep recurrent neural networks".

\section{Appendix: Philosophical Implications}

In the natural sciences, realism is often implicitly taken as true and, hence, little discussed. In philosophy, realism is a topic since ancient times and scepticism about objects is not new even for empiricists (Quine, 1980). Here, we quickly sketch the relationships between our perceptual approach and classic philosophical concepts to highlight important similarities and differences, thus, putting our ideas on a general landscape and making clear where we stand and, more importantly, where we do not stand. A detailed analysis of the modern discussion regarding ordinary objects (Korman, 2020; Ladyman et al., 2007; Merricks, 2003; Van Inwagen \& Van Inwagen, 1990) is forthcoming.

Realism and Idealism. Most philosophical theories either subscribe to a realism where objects are mind independent (Crane \& French, 2015; Descartes, 1637/1987; Hume, 1739/2003; Locke, 1689/1841; Moore, 1905) or to idealistic positions where objects disappear when they are not perceived (Berkeley, 1710/1881; Fichte, 1796/1998; Hegel, 1807/2012). We maintain an intermediate position: there is a mind independent world, described by the world vector, but objects are mind dependent. 
The non-uniqueness of objects may be taken as evidence for dualism, similarly to the ontological status of sense data (Ayer, 1942). However, because objects are mind dependent, the question vanishes. There are no sense data. Objects are equivalent to mental representations.

Platonism \& Ontology. Perception is a many-few mapping from a large set of physical states, described by the world vector, to a few representations, which tessellate the world vector into "invariants". Platonism is just the opposite: a few mind independent invariants create a large world of appearances. Modern physics may be seen as the search for laws, which determine the world vector. The laws, if they were once found, would not be in the external world but in the mind. If we could predict the world vector from an equation, we would still not know about percepts.

Kant. Our proposal is different from a Kantian position since we accept particle physics. We know about the ontology of the world and we can compute the states of the world in principle and to some extent also in practice, such as the reconstruction of the first milliseconds after the big bang. In addition, our position is not Kantian since we propose that the mental states of humans may strongly vary. At best one can find out only about one's own "categories".

Underdetermination. Our arguments are very close to the underdetermination argument in the philosophy of science (Duhem, 1991), which states that there can be many theories, which explain the same given set of data. In perception, two perceptual systems may have a large subset of identical percepts but differ in many others (see Part II: Concepts I).

Primary and secondary properties. Often it is proposed that certain properties are more "real" than other, for example, colours are less real (Hardin, 1993). We propose that there are no differences in the ontological status of properties. It might be that certain properties are more important from an evolutionary point of view. For example, solidity may be more important than colour for humans. However, there are no principled differences.

Meaning \& Partitions. One may argue that the meaning of the representation of grandmother is just the number of all possible views of grandmother or all corresponding states in the external world, similar to Frege's notion of extension (Frege, 1892/1948). First, meaning in this respect is subjective. Second, we cannot determine the meaning of both the states and the representation because we do not know the mapping from the states to the mental representations and vice versa.

Tokens \& types. Only physical states are tokens, with many tokens mapping onto one mental representation. Hence, mental representations are always types. An object is not a token, it is a type because it represents an entire class of external states. We perceive types not tokens.

The argument from illusion and sense data. In naïve or direct realism, we directly perceive objects. Naïve realism is often challenged by the argument from illusion. For example, a straight wooden skewer is put in a water glass. The skewer is not perceived as straight; it has a shift at the interface between the air and the water (Ayer, 1942; Hume, 2003). It is argued that we do not see the stick directly because otherwise we would see a straight skewer and, for this reason, we perceive some intermediate object, often called sense data. We agree that we do not see objects as they really are because there are no mind-independent objects and, hence, there are no mind-independent straight skewers. There is no veil of perception hiding the true appearance of an object because there are no mind-independent objects. There are no intermediate sense 
data. Perception is not about aboutness in the sense that percepts can be compared with something out there, they are not about anything mind-independent. In addition, percepts are not instances of the most prototypical view (see also Sheldon, 1985). We may say that the straight skewer represents the skewer better than the shifted one because it appears straight normallynot because this is the veridical view. However, this is a cognitive and not a perceptual issue.

Perceptual identity. Object representations give rise to the notion of identity because, for example, they link external world states across time by the activation of the same mental representation. Identity is similarity across time relative to how the system partitions the world state. There is no identity in the real world because the world states, which belong to the same object representation, differ from each other. In addition, "identity" varies across perceptual systems. The term identity should, hence, be used with care. For example, Leibniz's law of the indiscernibility of identicals cannot be applied to objects since, as just mentioned, an object stands for a class of different states, which in addition may change. Leibniz's law can only be used in mathematics and semantics (a bachelor is equal to an unmarried man).

Subjectivity. The classic notion of subjectivity is that only one-self has access to one's own percepts and thoughts (Davidson, 1984). A related but different notion is that perception is subjective because it is highly individual, i.e., even though the very same object is presented, different people may perceive it differently. We adopted the second notion. Subjectivity can obviously not be eliminated because perception is always subjective. It is its nature but not because of privacy.

As a side note, if realism were true, there would be no first-person authority because the worldmind correspondence would be the same for all humans and perceptual systems. The presentation of a red square leads to the perception of the very same red square for all observers and thus everyone would know about other people's percepts.

As a second side note. Subjectivity is only good in small doses. Had humans fundamentally different visual systems, communication would be largely impossible. As a hypothesis, communication with animals is not only impossible because animals have no elaborated language skills but also because they perceive the world very differently. As Wittgenstein (1953/2009) said: "If a lion could speak, we could not understand him".

Consciousness. Our arguments hold true for both conscious and unconscious perception.

Epistemology. The starting point of epistemology has always been the percepts of humans. However, this philosophical approach does not allow one to realize that there are, for example, many states giving rise to the same representation of an object. Likewise, perception itself cannot tell us that perception is ill-posed. In addition, epistemology has always operated with extremely simple scenarios, such as one object and one representation, which misses the complexity problems of perception. If the world was ontologically simple, we might indeed veridically perceive the few existing entities and their relations. There would be no dispute and no epistemology. We have discussions about truth because the world is large and hence perception not unique.

Truth. This contribution is about perception, about correspondence, and world-mind mappings. It is not about truth, which is about propositions. Perception is "what it is", it is neither true nor false. In a forthcoming article, we will show that it is impossible to ground a theory of truth on 
perceptual realism because perception is not "realistic". For this reason, truth can only be true in the context of a specific perceptual system, i.e., truth is reference frame dependent, or in other words mind dependent.

\section{References}

Ahissar, M., \& Hochstein, S. (1993). Attentional control of early perceptual learning. Proceedings of the National Academy of Sciences, 90(12), 5718-5722.

Anderson, B. L., \& Winawer, J. (2008). Layered image representations and the computation of surface lightness. Journal of Vision, 8(7), 18-18.

Ayer, A. J. (1942). The foundations of empirical knowledge.

Barlow, H. B. (1972). Single units and sensation: A neuron doctrine for perceptual psychology? Perception, 1(4), 371-394.

Barnes, B., Bloor, D., \& Henry, J. (1996). Scientific knowledge: A sociological analysis. University of Chicago Press.

Barron, A. B., \& Klein, C. (2016). What insects can tell us about the origins of consciousness. Proceedings of the National Academy of Sciences, 113(18), 4900-4908.

Berkeley, G. (1881). A treatise concerning the principles of human knowledge. JB Lippincott \& Company.

Blakemore, C., \& Cooper, G. F. (1970). Development of the brain depends on the visual environment. Nature, 228(5270), 477-478.

Bosten, J. M., \& Mollon, J. D. (2010). Is there a general trait of susceptibility to simultaneous contrast? Vision Research, 50(17), 1656-1664. 
Campos, J. J., Hiatt, S., Ramsay, D., Henderson, C., \& Svejda, M. (1978). The emergence of fear on the visual cliff. In The development of affect (pp. 149-182). Springer.

Caparos, S., Ahmed, L., Bremner, A. J., de Fockert, J. W., Linnell, K. J., \& Davidoff, J. (2012). Exposure to an urban environment alters the local bias of a remote culture. Cognition, 122(1), 80-85.

Cappe, C., Clarke, A., Mohr, C., \& Herzog, M. H. (2014). Is there a common factor for vision? Journal of Vision, 14(8), 4-4.

Churchland, P. M., \& Churchland, P. S. (1998). On the contrary: Critical essays, 1987-1997. MIT Press.

Crane, T., \& French, G. (2015). The Problem of Perception in n EN Zalta, ed. Stanford Encyclopaedia of Philosophy. http://plato. stanford. edu/entries ....

Daoudi, L. D., Doerig, A., Parkosadze, K., Kunchulia, M., \& Herzog, M. H. (2017). The role of oneshot learning in\# TheDress. Journal of Vision, 17(3), 15-15.

Davidson, D. (1984). First person authority. Dialectica, 38(2-3), 101-111.

de Fockert, J., Davidoff, J., Fagot, J., Parron, C., \& Goldstein, J. (2007). More accurate size contrast judgments in the Ebbinghaus Illusion by a remote culture. Journal of Experimental Psychology: Human Perception and Performance, 33(3), 738.

Descartes, R. (1987). La Dioptrique (1637). CEuvres et Lettres.

Descartes, R. (1996). Discourse on the method: And, meditations on first philosophy. Yale University Press.

Duhem, P. M. M. (1991). The aim and structure of physical theory (Vol. 13). Princeton University Press. 
Fahle, M., \& Morgan, M. (1996). No transfer of perceptual learning between similar stimuli in the same retinal position. Current Biology, 6(3), 292-297.

Fichte, J. G. (1998). Foundations of transcendental philosophy (Wissenschaftslehre) nova methodo (1796/99). Cornell University Press.

Field, T. M., Cohen, D., Garcia, R., \& Greenberg, R. (1984). Mother-stranger face discrimination by the newborn. Infant Behavior and Development, 7(1), 19-25.

Frank, M. C., Vul, E., \& Johnson, S. P. (2009). Development of infants' attention to faces during the first year. Cognition, 110(2), 160-170.

Freeman, J., \& Simoncelli, E. P. (2011). Metamers of the ventral stream. Nature Neuroscience, 14(9), 1195-1201. https://doi.org/10.1038/nn.2889

Frege, G. (1948). Sense and reference. The Philosophical Review, 57(3), 209-230.

Frith, C. (2013). Making up the mind: How the brain creates our mental world. John Wiley \& Sons.

Gibson, E. J., \& Walk, R. D. (1960). The" visual cliff.". Scientific American.

Griffith, T., \& Ferrari, G. R. F. (2000). Plato:'The Republic'. Cambridge University Press.

Grossberg, S. (2013). Adaptive Resonance Theory: How a brain learns to consciously attend, learn, and recognize a changing world. Neural Networks, 37, 1-47.

Grzeczkowski, L., Clarke, A. M., Francis, G., Mast, F. W., \& Herzog, M. H. (2017). About individual differences in vision. Vision Research, 141, 282-292.

Hardin, C. L. (1993). Color for Philosophers (expanded edition). Indianapolis: Hackett.

Hegel, G. W. F. (2012). The phenomenology of mind. Courier Corporation. 
Henrich, J., Heine, S. J., \& Norenzayan, A. (2010). Most people are not WEIRD. Nature, 466(7302), 29.

Herzog, M. H., \& Esfeld, M. (2009). How the mind constitutes itself through perceptual learning. Learning \& Perception, 1(1), 147-154.

Hoffman, D. (2019). The case against reality: Why evolution hid the truth from our eyes. WW Norton \& Company.

Hoffman, D. D., Singh, M., \& Mark, J. (2013). Does evolution favor true perceptions? Human Vision and Electronic Imaging XVIII, 8651, 865104.

Hofstadter, D., \& Sander, E. (2013). Surfaces and essences: Analogy as the fuel and fire of thinking. Basic Books.

Hornik, K., Stinchcombe, M., \& White, H. (1989). Multilayer feedforward networks are universal approximators. Neural Networks, 2(5), 359-366.

Hume, D. (2003). A treatise of human nature. Courier Corporation.

Ilyas, A., Santurkar, S., Tsipras, D., Engstrom, L., Tran, B., \& Madry, A. (2019). Adversarial examples are not bugs, they are features. ArXiv Preprint ArXiv:1905.02175.

Jacobsen, J.-H., Behrmann, J., Zemel, R., \& Bethge, M. (2018). Excessive Invariance Causes Adversarial Vulnerability. ArXiv Preprint ArXiv:1811.00401.

Kammer, T., Puls, K., Erb, M., \& Grodd, W. (2005). Transcranial magnetic stimulation in the visual system. II. Characterization of induced phosphenes and scotomas. Experimental Brain Research, 160(1), 129-140.

Kanizsa, G. (1979). Organization in vision: Essays on Gestalt perception. Praeger Publishers. Koenderink, J. (2014). The all seeing eye? SAGE Publications Sage UK: London, England. 
Koenderink, J., \& van Doorn, A. (2008). The structure of visual spaces. Journal of Mathematical Imaging and Vision, 31(2-3), 171.

Korman, D. Z. (2020). Ordinary Objects. In E. N. Zalta (Ed.), The Stanford Encyclopedia of Philosophy (Fall 2020). Metaphysics Research Lab, Stanford University. https://plato.stanford.edu/archives/fall2020/entries/ordinary-objects/ Ladyman, J. (2014). Structural realism. Stanford Encyclopedia of Philosophy. Ladyman, J., Ross, D., Spurrett, D., Spurrett, D., Collier, J., \& Collier, J. G. (2007). Every thing must go: Metaphysics naturalized. Oxford University Press on Demand.

Lafer-Sousa, R., Hermann, K. L., \& Conway, B. R. (2015). Striking individual differences in color perception uncovered by 'the dress' photograph. Current Biology, 25(13), R545-R546. Laudan, L. (1981). A confutation of convergent realism. Philosophy of Science, 48(1), 19-49. Leibowitz, H. W., \& Pick, H. A. (1972). Cross-cultural and educational aspects of the Ponzo perspective illusion. Perception \& Psychophysics, 12(5), 430-432.

Locke, J. (1841). An essay concerning human understanding.

Lotto, B. (2017). Deviate: The science of seeing differently. Hachette Books.

Luhmann, N. (1986). The autopoiesis of social systems. Sociocybernetic Paradoxes, 6(2), 172192.

Marr, D. (1982). Vision: A computational investigation into the human representation and processing of visual information. W.H. Freeman.

Mausfeld, R. (2015). Notions such as "truth" or "correspondence to the objective world" play no role in explanatory accounts of perception. Psychonomic Bulletin \& Review, 22(6), 1535-1540. 
Merricks, T. (2003). Objects and persons. Oxford University Press on Demand.

Moore, G. E. (1905). The nature and reality of objects of perception. Proceedings of the Aristotelian Society, 6, 68-127.

O’Regan, J. K., \& Noë, A. (2001). A sensorimotor account of vision and visual consciousness. Behavioral and Brain Sciences, 24(5), 939-973.

Popper, K. (2005). The logic of scientific discovery. Routledge.

Putnam, H. (1981). Brains in a Vat.

Reid, T. (1764). An Inquiry into the Human Mind (D. Brookes, Ed.). Edinburgh: Edinburgh.

Schwartz, R. (2016). Perceptual Veridicality. Philosophical Topics, 44(2), 381-404.

Searle, J. R. (2015). Seeing things as they are: A theory of perception. Oxford University Press.

Segall, M. H., Campbell, D. T., \& Herskovits, M. J. (1963). Cultural differences in the perception of geometric illusions. Science, 139(3556), 769-771.

Seth, A. (2021). Being you: A new science of consciousness. Penguin.

Sheldon, N. A. (1985). One wave or three? A problem for realism. The British Journal for the Philosophy of Science, 36(4), 431-436.

Smith, B. C. (1996). On the Origin of Objects. MIT Press.

Su, J., Vargas, D. V., \& Sakurai, K. (2019). One pixel attack for fooling deep neural networks. IEEE Transactions on Evolutionary Computation.

Szegedy, C., Zaremba, W., Sutskever, I., Bruna, J., Erhan, D., Goodfellow, I., \& Fergus, R. (2013). Intriguing properties of neural networks. ArXiv Preprint ArXiv:1312.6199.

Tanaka, J. W., \& Farah, M. J. (1993). Parts and wholes in face recognition. The Quarterly Journal of Experimental Psychology, 46(2), 225-245. 
Thompson, P. (1980). Margaret Thatcher: A new illusion. Perception.

Thorpe, S. J., \& Fabre-Thorpe, M. (2001). Seeking categories in the brain. Science, 291(5502), 260-263.

Todorovic, D. (2016). Is the Muller-Lyer illusion a perspective-based illusion? PERCEPTION, 45, 364-365.

Van Fraassen, B. C. (1980). The scientific image. Oxford University Press.

Van Inwagen, P., \& Van Inwagen, P. (1990). Material beings. Cornell University Press.

Vilares, I., \& Kording, K. (2011). Bayesian models: The structure of the world, uncertainty, behavior, and the brain. Annals of the New York Academy of Sciences, 1224(1), 22.

von Helmholtz, H. (1883). Wissenschaftliche abhandlungen (Vol. 2). Johann Ambrosius Barth. von Orman Quine, W. (1980). From a Logical Point of View: Nine Logico-philosphical Essays. Harvard University Press.

Wittgenstein, L. (2009). Philosophical investigations. John Wiley \& Sons.

Wolpert, D. H., \& Macready, W. G. (1997). No free lunch theorems for optimization. IEEE Transactions on Evolutionary Computation, 1(1), 67-82. 